\title{
Archiv der Pharmazie
}

302. Band, Heft 8, Seite 572-583

Verlag Chemie, GmbH, Weinheim / Bergstr.

W. Wiegrebe, E. Roesel und H. Budzikiewicz

Synthese des 6,7-Dimethoxy-3-(3,4-dimethoxy- $\alpha$-hydroxybenzyl)-2-methyl-1,2,3,4-tetrahydroisochinolins 
W. Wiegrebe, E. Roesel und H. Budzikiewicz

\section{Synthese des 6,7-Dimethoxy-3-(3,4-dimethoxy- $\alpha$-hydroxy- benzyl)-2-methyl-1,2,3,4-tetrahydroisochinolins*)}

Aus dem Institut für Pharmazeutische Technologie der Technischen Universität Braunschweig und dem Institut für Molekulare Biologie, Biochemie und Biophysik, Stöckheim b.Braunschweig

(Eingegangen am 9. Oktober 1968)

Die Synthese und das chemische Verhalten von 6,7-Dimethoxy-3-(3,4-dimethoxy- $\alpha$ hydroxybenzyl)-2-methyl-1,2,3,4-tetrahydroisochinolin (I) werden beschrieben.

\section{Synthesis of 6,7-Dimethoxy-3-(3,4-dimethoxy- $\alpha$-hydroxybenzyl)-2-methyl- 1,2,3,4-tetrahydroisoquinoline}

The synthesis and chemical reactivity of 6,7-dimethoxy-3-(3,4-dimethoxy- $\alpha$-hydroxybenzyl)-2-methyl-1,2,3,4-tetrahydroisoquinoline (I) are described.

6,7-Dimethoxy-3-(3,4-dimethoxy- $\alpha$-hydroxybenzyl)-2-methyl-1,2,3,4-tetrahydroisochinolin (I) wurde im Zusammenhang mit der Untersuchung über Umsetzungen des 1,2-Dihydro-2-methylpapaverinols in verdünnter Essigsäure synthetisiert $\left.{ }^{1}\right)$ : am Benzyl-C des 6,7-Dimethoxy-3-veratryl-isochinolins (II) (Darstellung vgl. ${ }^{2}$ )) wurde zuerst die Sauerstoffunktion eingeführt, danach wurde am Stickstoff quaternisiert und anschließend zu I reduziert.

Zunächst wurde versucht, durch Oxydation mit $\mathrm{Hg}$ (II)-acetat in essigsaurer Lösung analog Gadamer und Schulemann ${ }^{3}$ ) aus II direkt III zu erhalten. Da im Gegensatz zu Papaverin, das unter diesen Bedingungen zum entsprechenden Carbinol oxydiert wird, keine Reaktion eintrat, wurde versucht, II mit Selendioxid in Dioxan zu IV umzusetzen ${ }^{4}$ ). Auch diesmal blieb die Reaktion aus. Erst beim Erhitzen einer trockenen Mischung von Selendioxid und $\left.\mathrm{II}^{5}\right)^{6}$ ) konnte IV in etwa 40proz. Ausbeute erhalten werden.

*) 3. Mitt. über Dihydroisochinolyl-phenyl-carbinole.

2. Mitt. s. ${ }^{1}$.

1. Mitt. Arch. Pharmaz. 302, 22 (1969); das Reaktionsschema S. 24, unteres Drittel, enthält einen Setzfehler: die Pfeilspitzen sind so anzuordnen, daß der Zerfall von III in VI und VIII ersichtlich wird.

1) W. Wiegrebe und E. Roesel, Arch. Pharmaz. 302, 310 (1969).

2) W. Wiegrebe, D. Sasse und E. Roesel, Arch. Pharmaz. 301, 33 (1968).

8) J. Gadamer und W. Schulemann, Arch. Pharmaz. 253, 284 (1915).

4) R. S. Barrows und H. G. Lindwall, J. Amer. chem. Soc. 64, 2430 (1942).

5) J. J. Postowsky und B. P. Lugowski, Ber. dtsch. chem. Ges. 68, 852 (1935).

$\left.{ }^{6}\right)$ E. P. Taylor, J. Pharmacy Pharmacol. 2, 324 (1950). 
<smiles>[R]c1ccc(Cc2cc3cc([R])c([R])cc3cn2)cc1[R]</smiles>

II<smiles>[R]c1ccc(C(=O)c2cc3cc([R])c([R])cc3cn2)cc1[R]</smiles>

IV

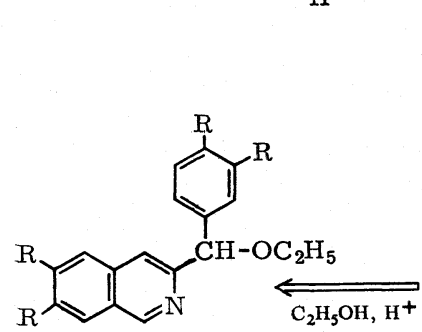

VIII

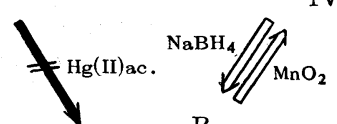<smiles>[R]c1ccc(C(O)c2cc3cc([R])c([R])cc3cn2)cc1[R]</smiles>

III<smiles>[R]c1ccc(C(O)c2cc3cc([R])c([Y])cc3c[n+]2C)cc1[R]</smiles>
$\mathrm{NaBH}_{4}$<smiles>[R]c1cc2c(cc1[R])CN(C)C(C(C)O)C2</smiles><smiles>CC</smiles>

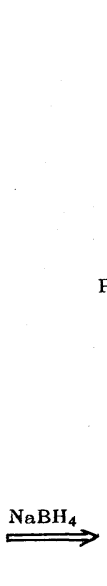<smiles>[R]c1ccc(C(=O)c2cc3cc([R])c([R])cc3c[n+]2C)cc1[R]</smiles>
$\mathrm{NaBH}_{4}$

VII<smiles>[R]c1ccc(C(=O)C2Cc3cc([R])c([R])cc3CN2C)cc1[R]</smiles>

$\mathrm{R}=\mathrm{OCH}_{3}$

Abb. 1

Durch Erhitzen von IV mit Methyljodid ${ }^{7}$ ) wurde $V$ erhalten, das bei der Reduktion mit Natriumborhydrid ${ }^{8}$ ) I als öliges Produkt lieferte (Abb. 1; einfacher Pfeil). Im DC zeigt I (Abb. $2 ; 2 \alpha$ und $2 \beta$ ) wie das Reduktionsprodukt ${ }^{1}$ ) der 3,4-Dihydro-isochinoliniumphase aus der Umsetzung von 1,2-Dihydro-papaverinol in verdünnter Essigsäure (Abb.2; $1 \alpha$ und $1 \beta$ ) zwei Flecke, die bei der Detektion mit Formaldehyd-Schwefelsäure die beschriebene Färbung ${ }^{1}$ ) zeigen.

7) G. Goldschmiedt, Mh. Chem. 7, 489 (1886).

$\left.{ }^{8}\right)$ E. Schenker, Angew. Chem. 73, 81 (1961). 


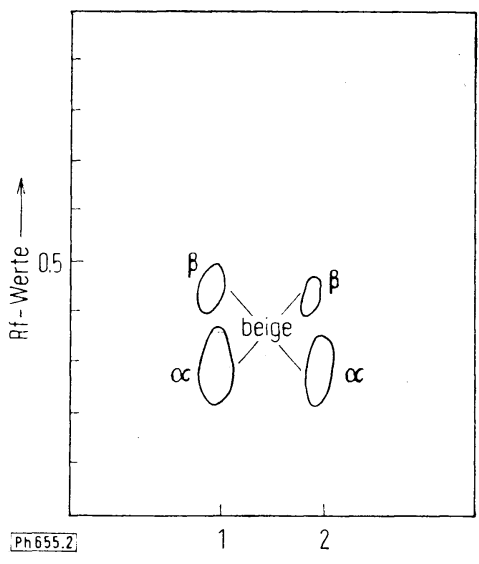

Abb. 2

Wir versuchten, I als Oxalat zu kristallisieren; es schieden sich jedoch nur ölige Massen $a b$, aus denen I zurückgewonnen wurde, das auf einer Kieselgelsäule nicht in die beiden Komponenten zu trennen war. Der Versuch, I als p-Nitroazobenzolcarbonsäureester ${ }^{9}$ ) kristallin $\mathrm{zu}$ erhalten, blieb ebenfalls ohne Erfolg. Da auch alle weiteren Versuche, ein durch Schmelzpunkt, Elementaranalyse und IR-Spektrum definiertes I-Derivat zu erhalten, fehlgeschlagen waren, sollte die Struktur von I durch ein Massenspektrum gesichert werden. Das Fragmentierungsverhalten von I bestätigt die erwartete Struktur (s. u.).

Neben I läßt sich im Massenspektrum 6,7-Dimethoxy - 3 - (3,4-dimethoxybenzoyl) - 2methyl-1,2,3,4-tetrahydroisochinolin (VI) erkennen. Für das Keton VI sind zwei Bildungsmöglichkeiten denkbar:

a) V wurde unvollständig reduziert; b) das Carbinol I wird zu VI oxydiert.

Wenn das Keton VI nach b) entsteht, so wäre es denkbar, I durch Oxydation mit aktivem Braunstein $^{10}$ ) in VI zu überführen. Beim entsprechenden Versuch wurde I in nicht definierter Weise gespalten.

Keiner der beiden Flecke im DC (Abb.2, $2 \alpha$ und $2 \beta)$, die auch nach katalytischer Hydrierung (vorhydriertes $\mathrm{PtO}_{2} / \mathrm{H}_{2} \mathrm{O}$ ) auftreten ${ }^{11}$ ) ${ }^{*}$ ), färbt sich beim Besprühen mit 2,4-Dinitrophenylhydrazin-Lösung an. Folglich kann keiner von beiden von dem Keton herrühren. Wir zogen daraufhin die Möglichkeit in Betracht, daß die beiden Flecke von Diasteromeren herrühren könnten.

Um eine unvollständige Reduktion der Carbonylfunktion auszuschließen und darüber hinaus die Möglichkeit zu schaffen, VII durch Salzbildung mit einer optisch aktiven Säure vor der Reduktion zu I in die optischen Antipoden zu spalten (s. u.), wurde I jetzt in der Weise synthetisiert, daß zuerst die Carbonylfunktion hydriert, danach am Stickstoff quaternisiert und dann zum Tetrahydroisochinolin reduziert wurde (Abb. 1, Doppelpfeil).

Die Reduktion von IV zu III gelang mit Natriumborhydrid ${ }^{8}$ ). III fällt als de einheitliches Öl an und wird in Kristallen erhalten, wenn die aus der Reduktion stammenden Verunreinigungen auf einer Kieselgelsäule abgetrennt werden. Bei dem Versuch, ein Pikrat herzustellen, wurde zwar ein kristallines Produkt erhalten, bei der dc Untersuchung der zurückgewonnenen Base stellte sich jedoch heraus,

*) Das UV-Spektrum von I (Abb. 5 a, s. u.) ist typisch für 1,2,3,4-Tetrahydroisochinolin-Verbindungen.

$\left.{ }^{9}\right)$ E. Hecker, Chem. Ber. 88, 1666 (1955).

10) J. Attenburrow, D. F. B. Cameron, J. H. Chapman, R. M. Evens, B. A. Hems, A. B.A. Jansen und T. Walker, J. chem. Soc. (London) 1952, 1094.

11) Organicum, 3. Auflage, S. 255, VEB Deutscher Verlag der Wissenschaften, Berlin 1964. 


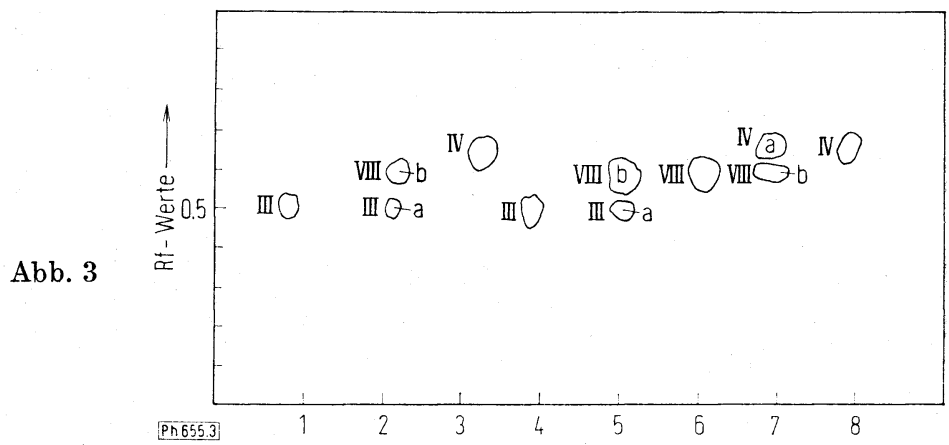

daß es sich jetzt um ein Gemisch aus zwei Dragendorff-positiven Stoffen handelt (Abb. 3, Flecke 2a und b; die römischen Zahlen neben den Flecken bezeichnen die zugehörigen Verbindungen in Abb. 1), die durch präparative Schichtchromatographie $\left.(\mathrm{PSC})^{12}\right)$ getrennt wurden. Beide Basen liefern nach dem Eluieren identische UVSpektren; NMR- und Massenspektrum zeigen, daß es sich bei der neu entstandenen Base um III-Äthyläther handelt $\left.{ }^{13}\right)$. Im NMR-Spektrum $\left(100 \mathrm{MHz}, \mathrm{CDCl}_{3}\right.$ als Lösungsmittel, TMS als innerer Standard) treten bei $\delta=3,62 \mathrm{ppm}$ das Quartett $(2 \mathrm{H})$ und bei $\delta=1,28 \mathrm{ppm}$ das Triplett $(3 \mathrm{H})$ des Äthoxyrestes auf. Auch die übrigen Signale stehen mit Struktur VIII im Einklang.

Das Massenspektrum ${ }^{14}$ ) von VIII (Abb. 4) zeigt das Molekülion (m/e 383), das $\mathrm{C}_{2} \mathrm{H}_{5} \cdot(\mathrm{m} / \mathrm{e} 354, c)$ sowie $\mathrm{C}_{2} \mathrm{H}_{5} \mathrm{O} \cdot(\mathrm{m} / \mathrm{e} 338, d)$ verliert. Der Basispeak (m/e 339,e) entsteht durch McLafferty-Umlagerung ${ }^{15}$ ) unter Verlust von Acetaldehyd.
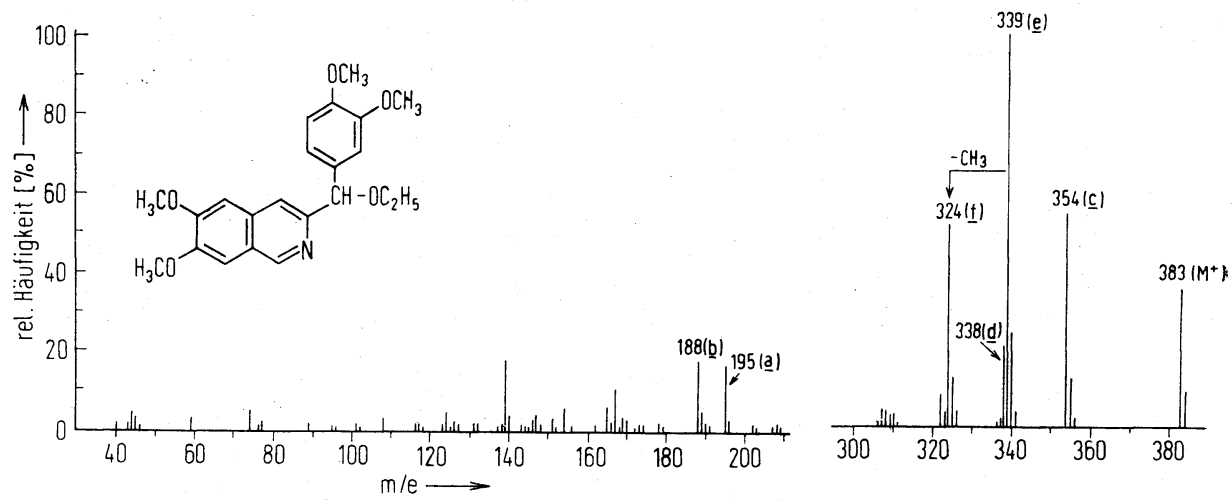

Abb. 4

12) H. Halpaap, Chemie-Ing.-Techn. 35, 488 (1963); Chemiker-Ztg. 89, 835 (1965).

13) W. Wiegrebe, Arch. Pharmaz. 300, 336 (1967).

$\left.{ }^{14}\right)$ H. Budzikiewicz, C. Djerassi und D. H. Williams, Mass Spectrometry of Organic Compounds, Holden-Day, Inc., San Francisco, 1967. Zur im Formelschema verwendeten Symbolik s. S. $1-4$.

15) ibid., S. 247. 
<smiles>COc1ccc([C+]c2cc3cc(OC)c(OC)cc3cn2)cc1OC</smiles>

$\mathrm{m} / \mathrm{e} 338(d)$<smiles>COc1cc2cnc(C=C3C=CC(=O)[C@@H](OC)C3)cc2cc1OC</smiles>

$\mathrm{m} / \mathrm{e} 324$ (f)

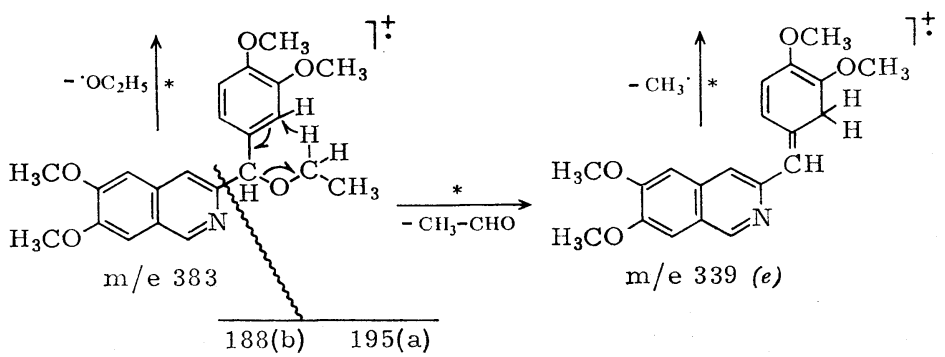<smiles>COc1ccc(C([OH2+])c2cc3cc(OC)c(OC)cc3cn2)cc1OC</smiles>

$\mathrm{m} / \mathrm{e} 354(c)$<smiles>COc1ccc([C@H](O)c2cc3cc(OC)c(OC)cc3cn2)cc1OC</smiles>

$\left(c^{\prime}\right)$

Das Fragment $e$ verliert - wie es bei methoxylierten Aromaten häufig zu beobachten ist $\left.{ }^{16}\right)$ - weiterhin ein Methylradikal (m/e 324, $f$ ).

Läßt man auf ein Gemisch von III und VIII (Abb. 3; Flecke 5a und 5b) aktives Mangandioxid ${ }^{\mathbf{1 0}}$ ) einwirken, so wird III (Abb.3, 5a) quantitativ zu IV (Abb.3, 7a) oxydiert, während VIII (Abb. 3, 5 b) erwartungsgemäß nicht verändert wird (Abb. 3, $7 \mathrm{~b})$. VIII läßt sich leicht wieder zerlegen. Nach 2stdg. Erwärmen in $\mathrm{HCl}$ ist der Äther quantitativ gespalten. Durch Erwärmen von III in absol. Äthanol unter Zusatz einiger Tropfen konz. Salzsäure konnte VIII kristallin erhalten werden. Da VIII sich leicht zersetzt, lieferte die Elementaranalyse kein zufriedenstellendes Ergebnis.

Das IR-Spektrum von III in Methylenchlorid zeigt, daß III frei von Keton ist. Die anfangs $0,05 \mathrm{~m}$ Lösung wurde stufenweise verdünnt und jedesmal erneut gemessen. Dabei zeigt sich, daß eine Wasserstoffbrückenbindung zwischen dem Stickstoff des Isochinolinringes und der $\mathrm{OH}$-Gruppe am Benzyl-C ausgeschlossen werden kann, denn im Bereich $\check{\nu} 3250\left[\mathrm{~cm}^{-1}\right]$ findet sich keine Bande; es herrscht ausschließlich Assoziation der Hydroxyl-

16) ibid., S. 240. 
gruppen. In konzentrierter Lösung überwiegt das polymer assoziierte $\mathrm{OH}$, breite Bande bei $\nu 3280-3560\left[\mathrm{~cm}^{-1}\right]$. Die Bande des dimeren Hydroxyls $\tilde{\nu} 3520-3550\left[\mathrm{~cm}^{-1}\right]$ hebt sich kaum von der Polymerenbande ab; deutlich erkennbar ist die scharfe Bande der freien $\mathrm{OH}-$ Gruppe bei $\tilde{\nu} 3600\left[\mathrm{~cm}^{-1}\right]$. Mit steigender Verdünnung tritt die Polymerenbande mehr und mehr zugunsten der beiden anderen zurück*).

Durch Erhitzen von III mit Methyljodid ${ }^{5}$ ) wurde VII $(\mathrm{X}=J)$ erhalten. Die Reduktion von VII mit Natriumborhydrid ${ }^{8}$ ) liefert I. Auch diesmal konnte I nicht kristallin erhalten werden und zeigte auf dem DC zwei Flecke. Das bestärkte unsere Vermutung, daß die beiden Flecke von Diastereomeren herrühren (siehe auch ${ }^{\mathbf{1}}$ )). Das UV-Spektrum (Abb. 5, b) ist identisch mit dem, das nach der katalytischen Hydrierung von $\mathrm{V}$ erhalten wurde Abb. 5, a).

Wir versuchten, VII in die optischen Antipoden zu spalten. Dieser Versuch blieb bislang ohne Erfolg, da VII mit (+)-Camphersulfonsäure $^{17}$ ) und Dibenzoyl-L-weinsäure ${ }^{17}$ ) nur als Racemat und mit anderen optisch aktiven Säuren gar nicht kristallisierte.

Um eine Isolierung der vermutlich diastereomeren Basen zu erreichen, wurden Versuche zur Trennung durch PSC ${ }^{12}$ ) unternommen. Wir verwendeten statt der üblichen Schichten aus Kieselgel $\mathrm{PF}_{254}$ eine Beschichtung mit einem Gemisch aus Kieselgel $\mathrm{HF}_{254}$ und $20 \%$ Zellulose MN 300 und erzielten auf diese Weise eine bessere Trennung. Von den auf einer solchen Platte getrennten und mit $2 \mathrm{n} \mathrm{HCl}$ eluierten Bändern wurden UV-Spektren gemessen. Die Spektren sind identisch (Abb. 5, c und d) und mit dem Spektrum des Diastereomerengemisches von I

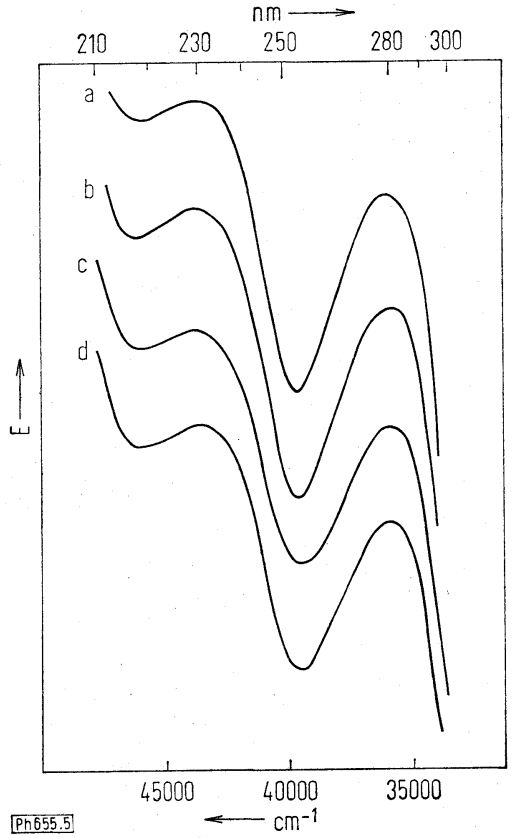

Abb. 5 (Abb.5, a und b) kongruent.

Die Schwierigkeit, I unzersetzt zu isolieren, liegt nach unseren Erfahrungen in der großen Empfindlichkeit begründet. I verträgt das Erwärmen mit $\mathrm{n} \mathrm{HCl}$, es tritt aber bereits Zersetzung ein, wenn man versucht, I aus saurer Lösung nach Ammoniakzugabe mit einem organischen Lösungsmittel auszuschütteln. Wir haben daher nach

*) Wir danken Herrn Prof. Dr.H.-H. Perkampus und Herrn Dr. H. Hoffmann für die Aufnahme und Diskussion des IR-Spektrums.

17) W.Theilacker in Houben-Weyl „Methoden der organischen Chemie“, 4. Aufl. Bd. 4/2 S. 516, Georg Thieme Verlag 1955. 
der Trennung der vermutlich diastereomeren Basen die Platte desaktiviert und die entsprechenden Bänder mit Benzol eluiert. Die Benzollösungen wurden auf ausgeglühte Kohle i. Vak. aufgezogen, um Massenspektren aufzunehmen. Die Spektren A und B (Abb. 6) sind sehr ähnlich. Da die Energieunterschiede stereoisomerer Verbindungen im allgemeinen zu klein sind, um im Massenspektrum mehr als qualitative Änderungen zu bewirken, stützen diese Spektren unsere Auffassung, daß die erwähnten zwei Flecke im DC von I auf Diastereomere zurückzuführen sind.
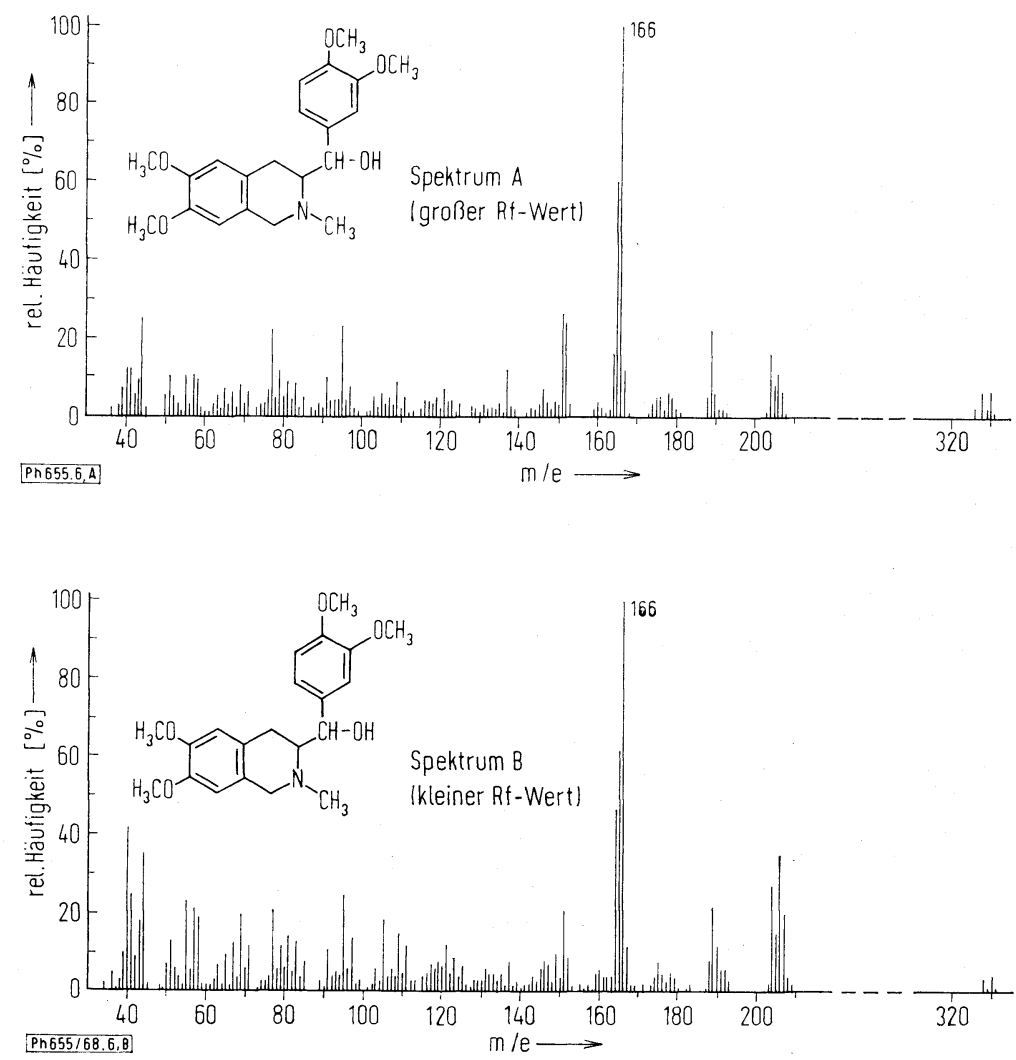

Abb. 6, A und B

Das ursprüngliche Massenspektrum war - wie ausgeführt - von einer I-Probe aufgenommen worden, die diesem Spektrum zufolge noch Keton VI enthielt. Wir haben daher das Massenspektrum (Spektrum C; Abb. 7) einer I-Probe gemessen, die durch Reduktion von VII gewonnen worden war und ketonfrei war. 


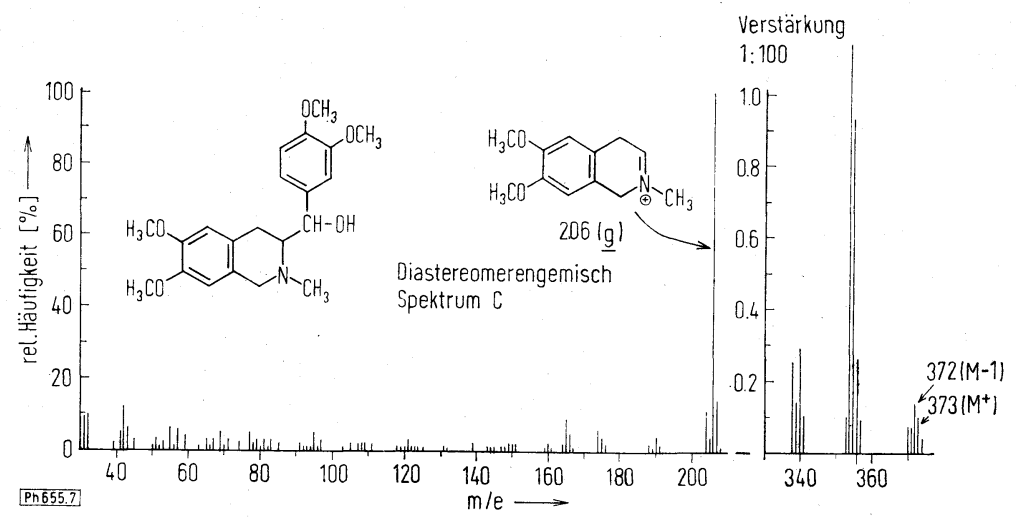

Abb. 7

Im Massenspektrum $\mathrm{C}$ des Diastereomeren-Gemisches tritt das Fragment des Dihydroisochinolinium-Ions (m/e 206, g) mit 100\% rel. Häufigkeit auf. Das Molekülion (m/e 373) ist erwartungsgemäß nur schwach, aber deutlich zu erkennen. Bei den nach der PSC gemessenen Spektren A und B ist das Molekülion nicht mehr vorhanden, dagegen ist freier Veratrumaldehyd (m/e 166), der im Spektrum C des Diasteromerengemisches nur in verschwindenden Mengen nachweisbar ist, hier mit $100 \%$ rel. Häufigkeit vertreten. Das für Spektrum C typische Fragment m/e 206 (g) tritt in den Spektren A und B nur mit 10 bis $35 \%$ rel. Häufigkeit auf, daneben ist auch noch das Spaltprodukt eines Dehydrierungsproduktes (aromatisierter Isochinolinium-Ring, m/e 204) in den Spektren A und B etwa gleich stark vertreten. Der Vergleich der Spektren A und B einerseits mit C läßt folgende Aussage zu:

Die Spektren A und B sind Mischspektren; die Proben enthalten freien Veratrumaldehyd. Die Tatsache, daß selbst unter den hier angewandten, sehr schonenden Operationen I bereits zersetzt wird, erklärt, weshalb es nicht gelang, I in kristalliner Form zu gewinnen.

Spektrum C steht mit der Struktur I im Einklang und beweist daher - unter Berücksichtigung der durch alle üblichen Methoden gesicherten Vorstufen - die Struktur des 6,7-Dimethoxy-3-(3,4-dimethoxy- $\alpha$-hydroxybenzyl)-2-methyl-1,2,3,4tetrahydroisochinolins für I.

Spektrum C ist darüber hinaus identisch mit dem Massenspektrum der durch Umlagerung aus 1,2-Dihydro-2-methylpapaverinol und nachfolgende Hydrierung entstandenen Verbindung und beweist damit die Struktur I auch für dieses Moleküli1). 


\section{Beschreibung der Versuche}

1. Oxydation von II mit $\left.\mathrm{SeO}_{2}{ }^{5}\right)^{6}$ )

$7 \mathrm{~g}$ II und $7 \mathrm{~g}$ frisch sublimiertes $\mathrm{SeO}_{2}{ }^{18}$ ) wurden $1 \mathrm{Std}$. im Metallbad von $190^{\circ}$ erhitzt. Die dunkle Reaktionsmasse wurde dreimal mit Methylenchlorid ausgekocht, dieses mit $10 \mathrm{~g}$ Kieselgel*) zur Trockne gebracht und auf eine Säule gegeben $(\varnothing 3,5 \mathrm{~cm}$; mit $700 \mathrm{~g}$ Kieselgel feucht gefüllt; Fließmittel: Aceton 1, Essigsäureäthylester 2). Die Fraktionen wurden de untersucht; nach dem Abdestillieren des Fließmittels wurde aus Benzol umkristallisiert. Ausbeute: $3 \mathrm{~g} \cong 41 \%$ d. Th.; Schmp. 192-193 ${ }^{\circ}$ (Benzol).

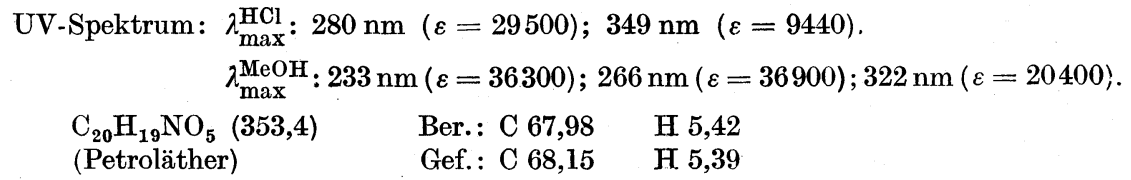

\section{Darstellung von $V^{?}$ )}

$1 \mathrm{~g}$ IV wurde mit überschüssigem Methyljodid im Bombenrohr 1 Std. auf $100^{\circ}$ erhitzt. Die gelben Nadeln von V wurden aus Wasser umkristallisiert. Ausbeute: $1,1 \mathrm{~g} \cong \mathbf{7 8 , 5} \%$ d. Th.; Schmp. 238-240 (Wasser).

$$
\begin{array}{lll}
\mathrm{C}_{21} \mathrm{H}_{22} \mathrm{NO}_{5} \mathrm{~J}(495,3) & \text { Ber.: C 50,92 } & \mathrm{H} \mathrm{4,48} \\
& \text { Gef.: C 51,24 } & \mathrm{H} \mathrm{4,27}
\end{array}
$$

\section{Reduktion von $\mathrm{V}$ zu I ${ }^{8}$ )}

Eine Lösung von $750 \mathrm{mg} \mathrm{V}$ in $75 \mathrm{ml}$ Lösungsmittel (Äthanol 2, Methylenchlorid 1, Wasser 1) wurde in kleinen Anteilen zu einer Anschlämmung von 1,5 g $\mathrm{NaBH}_{4}$ in $25 \mathrm{ml}$ Äthanol gegeben; beim Eintropfen färbte sich die Lösung tieforange, es wurde jedesmal Entfärbung abgewartet. Nachdem V zugegeben worden war, wurde mit weiteren $0,5 \mathrm{~g}$ $\mathrm{NaBH}_{4} 1$ Std. im Sieden gehalten. Nach dem Abkühlen wurde überschüssiges $\mathrm{NaBH}_{4}$ mit Eisessig zerstört und die Lösung i. Vak. auf etwa $25 \mathrm{ml}$ eingeengt. Nach Zugabe von $25 \mathrm{ml}$ Wasser wurde alkalisiert, mit Äther ausgeschüttelt und I als farbloses Öl erhalten. DC (Abb. 2): Sorptionsschicht: Kieselgel $\mathrm{HF}_{254}$; Fließmittel: Aceton 60, Methanol 25, Wasser 15; Laufstrecke $10 \mathrm{~cm}$; Detektion: UV-Licht $254 \mathrm{~nm}$, Formaldehyd-Schwefelsäure 30 Min. bei $120^{\circ}$.

\section{Säulenchromatographie von I}

$100 \mathrm{mg} \mathrm{V}$ wurden wie unter 3. beschrieben reduziert und aufgearbeitet. Die Ätherlösung wurde mit etwas Kieselgel zur Trockne gebracht und auf eine Säule gegeben. Mit Aceton 60, Methanol 25, Wasser 15 wurde eluiert. Die de Untersuchung zeigte, daß keine Trennung in die beiden Flecke eingetreten ist; es wurde auf I aufgearbeitet.

\section{Massenspektrum von I}

$50 \mathrm{mg} \mathrm{V}$ wurden wie üblich reduziert und aufgearbeitet; von dem ÖI wurde ein Massenspektrum gemessen. Neben I ist im Spektrum VI zu erkennen.

$$
\begin{array}{rrr}
\text { Mol.-Gew. I } & \text { Ber.: 373,1889 } & \text { Gef.: 373,1887 } \\
\text { VI } & \text { Ber.: 371,1733 } & \text { Gef.: 371,1730 }
\end{array}
$$

*) In allen Fällen wurde zur Säulenchromatographie Kieselgel 0,05-0,2 mm d. Fa. Merck verwendet.

18) Organicum, 3. Auflage, S. 593, VEB Deutscher Verlag der Wissenschaften, Berlin 1694. 


\section{Oxydation von $\mathrm{I}$ mit aktivem $\mathrm{MnO}_{2}{ }^{10}$ )}

$50 \mathrm{mg} \mathrm{V}$ wurden in üblicher Weise reduziert und aufgearbeitet. Die i. Vak. stark eingeengte Äther-Lösung wurde mit $10 \mathrm{ml}$ absol. Aceton und $300 \mathrm{mg}$ aktivem $\mathrm{MnO}_{2}$ versetzt. Nach 5stdg. Schütteln wurde filtriert und de untersucht; es war Spaltung von I eingetreten.

\section{Katalytische Hydrierung von $\mathrm{V}^{11}$ )}

$100 \mathrm{mg} \mathrm{V}$ wurden mit frisch gefälltem $\mathrm{AgCl}$ ins Chlorid überführt. Die wäßrige Lösung wurde i. Vak. eingeengt, mit $10 \mathrm{mg}$ vorhydriertem $\mathrm{PtO}_{2}$ versetzt und bei $20^{\circ}$ und Normaldruck bis zur Beendigung der $\mathrm{H}_{2}$-Aufnahme hydriert. Nach dem Abfiltrieren des Katalysators und Alkalisieren der wäßrigen Lösung wurde mit Äther ausgeschüttelt. Die getrocknete Ätherphase wurde eingeengt und dc untersucht. Wieder wurden die beiden Flecke erhalten (Abb. 2, $2 \alpha$ und $2 \beta$ ), keiner der beiden Flecke färbte sich mit 2,4-Dinitrophenylhydrazinlösung an. UV-Spektrum (Abb. 5, a): $\lambda_{\max }^{\mathrm{HCl}}: 230 ; 280 \mathrm{~nm}$.

\section{Reduktion von IV mit $\mathrm{NaBH}_{4}{ }^{8}$ )}

$3,5 \mathrm{~g}$ IV wurden in $240 \mathrm{ml}$ Äthanol gelöst, zu einer Anschlämmung von $0,7 \mathrm{~g} \mathrm{NaBH}_{4}$ in $10 \mathrm{ml}$ Äthanol gegeben und $1 \mathrm{Std}$. im Sieden gehalten. Die filtrierte Reaktionslösung wurde i. Vak. zur Trockne gebracht und der Rückstand 3mal mit $20 \mathrm{ml}$ Methylenchlorid ausgezogen. Die Lösung wurde mit $3 \mathrm{~g}$ Kieselgel zur Trockne gebracht und auf eine Säule gegeben $(\varnothing 2,3 \mathrm{~cm} ; 170 \mathrm{~g}$ Kieselgel feucht eingefüllt; Fließmittel: Aceton 65, Essigsäureäthylester 35). Die Fraktionen wurden de untersucht (Kieselgel $\mathbf{H F}_{254}$; Aceton 30, Chloroform 70) und stark eingeengt. III schied sich kristallin aus und ließ sich am Extraktor aus Äther umkristallisieren. Ausbeute: $2,44 \mathrm{~g} \bumpeq 69,5 \% \mathrm{~d}$. Th.; Schmp.: 161-163 ${ }^{\circ}$ (Äther).

$$
\begin{array}{lll}
\mathrm{C}_{20} \mathrm{H}_{21} \mathrm{NO}_{5}(355,4) & \text { Ber.: C 67,59 } & \text { H } 5,96 \\
& \text { Gef.: C 67,43 } & \text { H 6,04 }
\end{array}
$$

UV-Spektrum: $\lambda_{\max }^{\mathrm{MeOH}}: 240 ; 315 ; 327 \mathrm{~nm}$; Schulter bei $278 \mathrm{~nm}$.

$$
\lambda_{\max }^{\mathrm{HCl}}: 254 ; 310 \mathrm{~nm} \text {; Schulter bei } 280 \mathrm{~nm} \text {. }
$$

\section{Pikratbildung}

III wurde in Äthanol aufgenommen und mit äthanol. Pikrinsäurelösung versetzt. Das ausgefallene Pikrat wurde wiederholt aus Äthanol umkristallisiert; das Schmelzintervall änderte sich mit jedem Umkristallisieren. Das Pikrat wurde in Wasser gelöst und die stark alkalisch gemachte Lösung mit Äther ausgeschüttelt. Die gelbe Ätherlösung wurde mit 10proz. Kalilauge farblos gewaschen und chromatographiert.

DC: Sorptionsschicht: Kieselgel $\mathrm{GF}_{254}$; Fließmittel: Aceton 1, Essigsäureäthylester 1; Laufstrecke $10 \mathrm{~cm}$; Detektion: UV-Licht $254 \mathrm{~nm}$, Dragendorff's Reagens, FormaldehydSchwefelsäure $30 \mathrm{Min}$. bei $120^{\circ}$; Abb. 3: die Flecke 1 und 3 entsprechen III und IV Vergleichssubstanz, die Flecke a und b über Startpunkt 2 dem untersuchten Basengemisch.

\section{Präparative Schichtchromatographie ${ }^{12}$ )}

Eine wäßrige Lösung des Pikrates wurde stark alkalisch gemacht, mit Äther ausgeschüttelt und der getrocknete Äther abgedampft. Der Rückstand wurde in einem Gemisch Benzol 65, Essigsäureäthylester 35 aufgenommen und auf eine 40-cm-Platte aufgetragen (Schichtdicke $2 \mathrm{~mm}$ ). Es wurde $4 \mathrm{mal} \mathrm{mit} \mathrm{dem} \mathrm{genannten} \mathrm{Gemisch} \mathrm{entwickelt,} \mathrm{die} \mathrm{Bänder}$ wurden im UV-Licht $254 \mathrm{~nm}$ markiert und nach dem Abheben mit Aceton in der Kälte eluiert. Das Aceton wurde abgedampft, der Rückstand in $2 \mathrm{n} \mathrm{HCl}$ aufgenommen und nach 
dem Alkalisieren mit Äther ausgeschüttelt. Von dem Rückstand wurden ein NMR- und ein Massenspektrum (Abb. 4) gemessen. Das UV-Spektrum ist identisch mit dem von III, $\lambda_{\max }^{\mathrm{HCl}}: 252 ; 310 \mathrm{~nm}$.

\section{Oxydation von III mit aktivem $\mathrm{MnO}_{2}{ }^{10}$ )}

Ein Gemisch von III und VIII wurde in absol. Aceton 7 Std. mit aktivem $\mathrm{MnO}_{2}$ geschüttelt. Nach dem Filtrieren wurde die Acetonlösung eingeengt und dc untersucht, Abb. 3. Sorptionsschicht usw. wie unter 9.

\section{Darstellung von VIII}

III wurde in absol. Äthanol unter Zusatz einiger Tropfen konz. Salzsäure gelöst und 30 Min. auf dem Wasserbad erwärmt. Das Äthanol wurde i. Vak. abgezogen, der Rückstand in Wasser aufgenommen und nach dem Alkalisieren mit Äther ausgeschüttelt. Aus der getrockneten Ätherphase kristallisierte VIII nach dem Einengen; Schmp. 135 (Äther).

$$
\begin{array}{lll}
\mathrm{C}_{22} \mathrm{H}_{25} \mathrm{NO}_{5}(383,4) & \text { Ber.: C 68,91 } & \mathrm{H} 6,57 \\
& \text { Gef.: C 67,32 } & \mathrm{H} 6,46
\end{array}
$$

\section{Darstellung von $\left.V I^{7}\right)$}

1,5 g III wurden mit $5 \mathrm{ml}$ Methyljodid im Bombenrohr 1 Std. auf $100^{\circ}$ erhitzt und die gelben Nadeln aus Aceton umkristallisiert. Ausbeute: 1,84 仓 88\% d. Th.; Schmp. 174 bis $180^{\circ}$ (Aceton). IR-Spektrum: keine Bande im Carbonylbereich.

$$
\begin{array}{lll}
\mathrm{C}_{21} \mathrm{H}_{24} \mathrm{NO}_{5} \mathrm{~J}(497,3) & \text { Ber.: C 50,72 } & \mathrm{H} \mathrm{4,86} \\
& \text { Gef.: C 50,76 } & \mathrm{H} \mathrm{4,94}
\end{array}
$$

\section{Reduktion von VII zu $I^{8}$ )}

$100 \mathrm{mg}$ VII wurden mit $40 \mathrm{mg} \mathrm{NaBH}_{4}$ in Äthanol reduziert. Es wurde in üblicher Weise aufgearbeitet. Im DC 2 Flecke; UV-Spektrum (Abb. 5b): $\lambda_{\max }^{\mathrm{HCl}}: 230 ; 278 \mathrm{~nm}$.

\section{Darstellung des VII-Camphersulfonates ${ }^{17}$ )}

$1 \mathrm{~g}$ VII wurde in einer Mischung aus $15 \mathrm{ml}$ Wasser und $5 \mathrm{ml}$ Äthanol gelöst und mit der Lösung von $0,75 \mathrm{~g}$ Silbersalz der (+)-Camphersulfonsäure in $5 \mathrm{ml}$ Wasser versetzt. Vom ausgeschiedenen Silberjodid wurde abfiltriert und die Lösung i. Vak. eingeengt. Das VIICamphersulfonat schied sich in Kristallen vom Schmp. 125-128 aus. Theoretische Ausbeute: 0,6 g Salz für jeden der optischen Antipoden; gefunden wurden 0,79 g Salz (ohne Berücksichtigung der Mutterlauge). Das Salz wurde auf rac. VII aufgearbeitet, da auch durch fraktionierte Kristallisation keine Anreicherung eines Antipoden der Base zu erzielen war.

\section{Darstellung von dibenzoylweinsaurem VII ${ }^{17}$ )}

$512 \mathrm{mg} \mathrm{VII} \bumpeq 400 \mathrm{mg}$ Kation wurden unter Erwärmen in $20 \mathrm{ml}$ Äthanol gelöst und über einen $\mathrm{OH}$-beladenen Ionenaustauscher gegeben. Das Elut wurde mit der Lösung von $415 \mathrm{mg}$ Dibenzoyl-L-weinsäure in $1 \mathrm{ml}$ Äthanol versetzt. Es schieden sich Kristalle vom Schmp. $149-150^{\circ}$ aus. Theoretische Ausbeute: $375 \mathrm{mg}$ Salz für jeden der optischen Antipoden, gef.: $440 \mathrm{mg}$. $100 \mathrm{mg}$ Salz wurden in einer Mischung aus gleichen Teilen Wasser und Äthanol gelöst und über einen Cl-beladenen Ionenaustauscher gegeben. Das Elut wurde i. Vak. stark eingeengt und polarisiert; es war keine Drehung zu beobachten. 


\section{PSC von I}

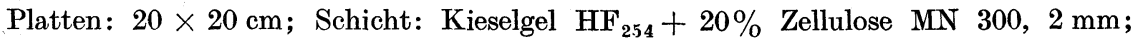
30 Min. bei $120^{\circ}$ aktiviert. Fließmittel: Aceton 60, Methanol 25, Wasser 15.

a) $50 \mathrm{mg}$ VII wurden wie üblich reduziert und aufgearbeitet. Die eingeengte Ätherlösung wurde bandförmig auf eine Platte aufgetragen und diese 3mal entwickelt. Die unter dem UV-Licht markierten Bänder wurden abgehoben und mit $2 \mathrm{n} \mathrm{HCl}$ eluiert. Von jedem Band wurde ein UV-Spektrum gemessen, Abb. $5 \mathrm{c}$ und d. $\lambda_{\max }^{\mathrm{HCl}}: 229 ; 278 \mathrm{~nm}$.

b) Eine weitere Platte wurde wie unter a) beschrieben angefertigt. Die Bänder wurden abgehoben, das Kieselgel mit $4 \mathrm{Tr}$. 10proz. Ammoniak-Lösung und $2 \mathrm{ml}$ Wasser desaktiviert und $4 \mathrm{Min}$. mit $10 \mathrm{ml}$ Benzol im Wasserbad erhitzt. Die benzolischen Lösungen wurden noch warm durch eine 3 G 5 M Fritte filtriert, i. Vak. auf ausgeglühte Kohle gezogen und Massenspektren gemessen, Abb. 6, Spektren A und B.

\section{Massenspektrum des I-Diastereomerengemisches}

$30 \mathrm{mg}$ VII wurde wie üblich reduziert und aufgearbeitet. Die getrocknete Ätherlösung wurde auf ausgeglühte Kohle gezogen und ein Massenspektrum gemessen; Spektrum C, Abb. 7.

\section{Allgemeine Angaben}

Die Schmp. wurden auf dem Kofler-Mikroheiztisch bestimmt und sind unkorrigiert. Die UV-Spektren wurden im Zeiss-Spektralphotometer PMQ II gemessen, die Massenspektren mit dem Gerät MS 9 der Fa. $A E I$ aufgenommen. Die IR-Spektren wurden im Perkin-Elmer-Gerät 521 in Methylenchlorid gemessen, das NMR-Spektrum wurde mit dem Varian-Gerät HA 100 registriert.

Anschriften: Doz. Dr. W. Wiegrebe, 33 Braunschweig, Pockelsstr. 4.

Doz. Dr. H. Budzikiewicz, 3301 Stöckheim b. Braunschweig, Mascheroder Weg 1. [Ph 655]

(c) Verlag Chemie GmbH, Weinheim/Bergstr., 1969

Bei der Wiedergabe von Gebrauchsnamen, Handelsnamen, Warenbezeichnungen u. dgl. handelt es sich häufig um gesetzlich geschützte eingetragene Warenzeichen, auch wenn sie nicht

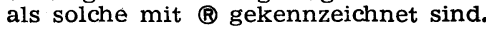

DG 5000/47/64 - III-18-127 